\title{
Continuous Distribution of Emission States from Single CdSe/ZnS Quantum Dots Supplementary Information
}

\author{
Kai Zhang ${ }^{1}$, Hauyee Chang ${ }^{1 \ddagger}$, Aihua $\mathrm{Fu}^{1}$, A. Paul Alivisatos ${ }^{1,2 *}$, and Haw Yang ${ }^{1,3 *}$ \\ ${ }^{1}$ Department of Chemistry, University of California at Berkeley and \\ ${ }^{2}$ Materials Sciences Division and ${ }^{3}$ Physical Biosciences Division, \\ Lawrence Berkeley National Laboratory, Berkeley, CA 94720
}

\section{EXPERIMENTAL}

\section{Functionalization of coverslips}

Glass coverslips (Fisher Scientific, 12-544-12) were cleaned by sonication in a 1-M potassium hydroxide solution followed by sonication in absolute ethanol for 15 mins each. Following each sonication step, the coverslips were thoroughly rinsed in filtered water (Nanopure Diamond, Barnstead). After repeating this procedure twice, water was removed from the coverslips by rinsing in acetone. The dried, clean coverslips were immediately immersed in a solution of $2 \%(\mathrm{v} / \mathrm{v})(3-$ aminopropyl)triethoxysilane (Aldrich, 440140) in acetone for 5 minutes. Excess silanization reagents on the surfaces were removed by washing with filtered water before the coverslips were cured at $110{ }^{\circ} \mathrm{C}$ for $30 \mathrm{~min}-$ utes. These steps introduced primary amine groups onto coverslip surfaces. Biotin-polyethylene glycol (biotinPEG-NHS, MW 3,400, 0H4M0F02, Nektar Therapeutics) and polyethylene glycol (mPEG-NHS, MW 5000, 2M4M0H01, Nektar Therapeutics) both containing the amine-reactive succinimidyl ester groups (-NHS) were used to functionalize the cover slip surfaces. $100 \mu \mathrm{L}$ solution of biotin-PEG-NHS (100 mg/mL) and PEG-NHS (ratio of 1:100) in $0.1 \mathrm{M} \mathrm{NaHCO} 3(\mathrm{pH} 8)$ was sandwiched between 2 coverslips for 3 hours for the reaction of the succinimidyl group with the primary amine groups. After incubation, excess PEG reagents were removed by repeated rinsing with filtered water.

\section{Quantum dots and their immobilization on coverslips}

The stock solution of QDs was diluted $10^{5}$ times in 0.5X Tris-borate-EDTA (TBE) buffer $(\mathrm{pH}=8.0) .20$ $\mu \mathrm{L}$ of the diluted solution was incubated for 10 minutes on a functionalized microscope cover slip. The sample was then washed with filtered water and another 20 $\mu \mathrm{L}$ of $0.5 \mathrm{X}$ TBE buffer was added to the sample, which was subsequently covered with a Coverwell (Molecular Probes). Coverage of QDs on the surface was checked

\footnotetext{
$\ddagger$ Current address: Xradia Inc., Concord, CA.
}

using our home-built single-molecule microscope and the dilution factor of the solution from the original QD stock was adjusted until an average density of $\sim 10$ QDs per $10 \times 10 \mu \mathrm{m}^{2}$ was achieved.

\section{Time-resolved single-molecule spectroscopy}

A schematic configuration for single-molecule optical experiments is shown in the main text. The $\sim 70$-fs excitation pulses were generated by a tunable Ti:sapphire oscillator (Spectra-Physics, Tsunami), which was pumped by a $10-\mathrm{W}$ frequency-doubled diode laser (Spectra-Physics, Millenia Xs). As the luminescence lifetime of QDs are relatively long, a Pockel cell (Conoptics, 350-160) was used to select for every 32nd pulse, increasing the inter-pulse duration from $12.5 \mathrm{~ns}$ to 400 ns. The Ti:sapphire oscillator was tuned to $980 \mathrm{~nm}$ and frequency doubled after the Pockel cell by a typeI $\beta-\mathrm{BaB}_{2} \mathrm{O}_{4}$ crystal (Casix). The beam was then recollimated and expanded to slightly under-fill the back aperture of an infinity-corrected microscope objective (Olympus, PlanApo, 60X, N.A. 1.4, oil immersion) inside a home-built, inverted confocal microscope. A 1/4 wave plate was placed in the beam path before the microscope to ensure a circularly polarized excitation. The incident light was directed through the objective via a long-pass dichroic filter (Chroma, Z488RDC) and focused onto a diffraction-limited spot. The mean incident excitation power at the sample was $28 \mathrm{~W} / \mathrm{cm}^{2}$. The luminescence photons from individual QDs were collected by the same objective and focused onto a single-photon counting avalanche photodiode module (APD, Perkin-Elmer, SPCM-AQD-14, 400-ps response time). Residual excitation light was removed by an dielectric emission filter (Chroma, HQ580LP) placed immediately before the APD. The emission filter was designed to have a spectral cutoff at $\sim 578 \mathrm{~nm}$, about $\sim 77 \mathrm{~nm}$ away from the QD emission peak at $655 \mathrm{~nm}$. Therefore, experimental observations of emission intensity were not affected by potential spectral diffusion of $\pm 10 \mathrm{~nm}[1,2]$. The dichroic filter and dielectric mirror inside the microscope may introduce variations in the detected photon flux for different emission polarizations. For example, CdSe nanorods with an aspect ratio of $\sim 2$, as that seen in the sample used in this experiment, have been shown to exhibit polarized emission [3]. The intensity variation due to different emission 
polarization was characterized and found to be less than $10 \%$.

The sample coverslip was secured onto a 2-D piezoelectric stage (Physik Instrumente, P731) via a vacuum chuck. The piezoelectric stage was steered by a high-voltage driver (Physik Instrumente, E509.C2 and E503.00) controlled by a computer through a multifunction I/O card (National Instruments, 6052E). Individual QDs were located by raster scanning the stage. Each of the randomly selected QDs was moved into the focus of the objective and time-resolved measurements of luminescence intensity of were made. Data was collected for at least 10 minutes for each QD. The average photoncounting rate was $\sim 3000$ counts per second (cps), well within the single photon counting regime [4].

The luminescence lifetime and intensity of individual QDs were measured using a time-correlated singlephoton counting (TCSPC) card (Becker\&Hickl, SPC630). For each detected photon, both the chronological arrival time and the time lapse between the excitation pulse and the emission photon (microtime) were recorded . The resolution for the photon arrival time is $\sim 150 \mathrm{~ns}$, determined by the dead time of the APD and the TCSPC card; this is the time resolution for inter-photon duration shown in the main text. The resolution of microtime was $\sim 1.56 \mathrm{~ns}$, determined by uniform digitization of 256 channels over the $400-n$ s repetition period between excitation pulses. To ensure that variations in the QD PL intensity does not result from laser fluctuations during the long experimental time, control experiments were performed immediately before and after each experimental session. Autocorrelation analysis confirmed that trajectories taken in the absence of QD sample showed no correlation.

As a control, experiments on a system that is known to exhibit a two-state behavior were carried out and data analyzed. Attenuated light was directly sent into an APD with a shutter in the light path. Chronological photon arrival time was recorded by TCSPC card and trajectories were reconstructed. In this configuration, the intensity states are designated as 'on' when the light pass through the shutter and 'off' when it is blocked. The results validate that the analysis indeed recognized and assigned two different intensity states. Intentity trajectories were taken with a shutter open/closed at $1 \mathrm{~Hz}$ in the light path. A representative 10-s trajectory is shown in Fig. 1. Changepoint analysis and BIC-grouping algorithm were first used to find the most probable number of intensity states for this trajectory. It was found that this model trajectory should indeed be dectibed by a twostate model. Next, the Kolmogorov-Smirnov (KS) test [5] was applied along the trajectory with the statistics computed every 10,000 photons or less. Using this test, the hypothesis that the observations are from an exponential distribution is rejected if any of the 10,000-photon section fails the test; the hypothesis is accepted if and only if all of the sections pass the test. The KS-test results for the control experiment is summarized in Table I, showing that the inter-photon duration from each of the BIC-grouped states is indeed exponentially distributed.

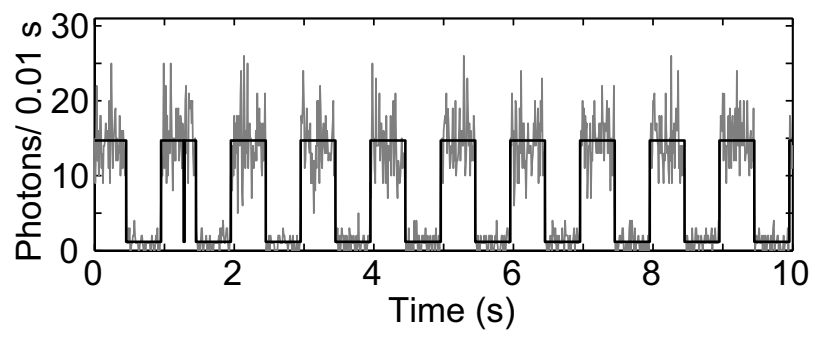

FIG. 1: A 10-second trajectory which is generated by setting the shutter open and close at $1 \mathrm{~Hz}$ with constant laser illumination. The raw photon arrival time data is binned at $10 \mathrm{~ms}$ (gray), on which the reconstructed trajectory from the changepoint and BIC-grouped analysis are overlaid (thick solid line).

TABLE I: Kolmogorov-Smirnov test of exponentiality for sections containing 10,000 photons or less in the time trajectories. $N$ is the total number of photons found in each state, $n$ is the number of photons used in each exponentiality test, $I$ is the average intensity of that state, $\mathrm{C} 1$ and $\mathrm{C} 2$ are respectively the high- and low-intensity states for the control experiment where a shutter is used to produce two well controlled intensity states. These assignments are based on the BIC-grouped analysis. The hypothesis that the observations are from an exponential distribution is rejected if $D$ exceeds the critical value, $\mathcal{E}_{0.01}$ for any of the section along the trajectory.

\begin{tabular}{crrrrr}
\hline \hline State & $N$ & $n$ & $I(\mathrm{cps})$ & $D$ & $\mathcal{E}_{0.01}$ \\
\hline $\mathrm{C} 1$ & 82033 & 10000 & 1470 & 0.0082 & 0.0125 \\
$\mathrm{C} 2$ & 6229 & 6229 & 117 & 0.0094 & 0.0158 \\
\hline
\end{tabular}

\section{STATISTICAL ANALYSIS OF INFORMATION-LIMITED SINGLE-MOLECULE DATA}

To visualize time-dependent emission intensity from a single QD, typically the number of photons detected within certain time interval is displayed as a function of chronological time. The width of each bin determines the time resolution of such a binned intensity trajectory. Fig. 2 shows a section of typical QD trajectory. The 1ms binned trajectory appears extremely noisy. This is because the intensity is inundated with Poisson counting noise when using such a small bin time. A longer bin time reduces the noise, but at the expense of worsening time resolution. Changes that occur on a time scale shorter than the bin widths, however, become unobservable or obscured. This is illustrated in by the 1-ms and 100-ms 
panels where, for example, the fast intensity transition occurring at $\sim 37.2 \mathrm{~s}$ of the 1-ms panel becomes merged with another transition under 100-ms binning.

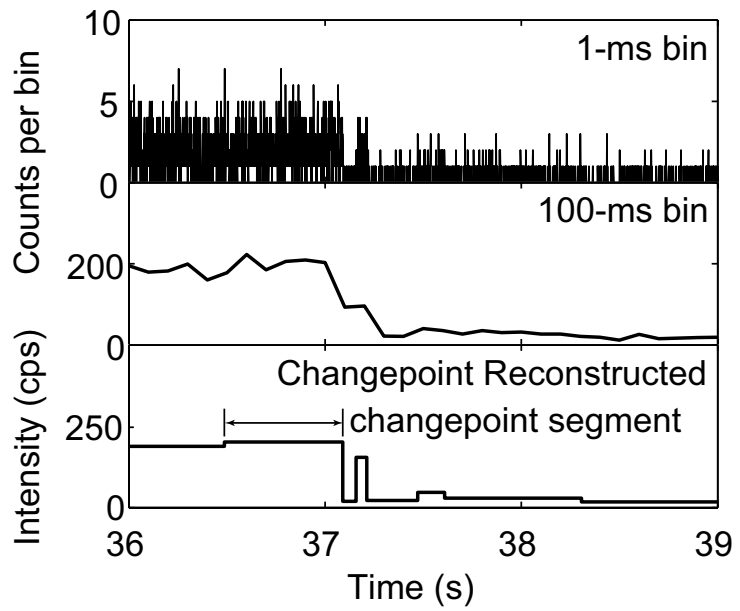

FIG. 2: A 3-second section of time-resolved single QD trajectory. The fast transition missed by the $100-\mathrm{ms}$ binned trajectory is resolved in the reconstructed trajectory using the changepoint algorithm (bottom panel). Subtle changes with long-term segments missed in both binned trajectories are also resolved. Here, a changepoint segment is defined as the photon stream that have chronological time stamps between the two sudden intensity jumps as indicated in the figure.

High time-resolution single-molecule spectroscopy through photon-by-photon reconstruction of intensity time trajectory

A statistically robust method for quantitatively reconstructing the underlying dynamics from a noisy singlemolecule intensity trajectory has been developed recently [6]. This technique, hereafter referred as the changepoint method, efficiently extracts information by operating on counting data photon by photon. It does not arbitrate bin widths in resolving intensity changes, nor does it involve artificial assignments to thresholds in determining intensity states. Further, the reconstruction of the underlying dynamics does not require any kinetic model. The basic assumption is that the time scale for the intensity state change is much faster than the experimental time resolution. This is complementary to another model-free method - the maximum-information method - that deals with cases where time scale of state changes is comparable to or slower than experimental time resolution [7]. Such model-free approaches allow in-depth studies of complex dynamics objectively.

In the changepoint method, the intensity change points are identified solely from the inter-photon time intervals using a generalized likelihood ratio (GLR) test. In essence, it compares the likelihood of having a sudden emission intensity change at the $m$-th photon, with that of having uniform intensity along a stream of $n$ photons:

$\mathrm{GLR}=\max _{1 \leq m \leq n}\left\{\frac{L(1 \ldots m \mid i) L(m+1 \ldots n \mid i+1)}{L(1 \ldots n \mid i)}>\mathcal{C}\right\}$,

where $L(1 \ldots m \mid i)$ denotes the likelihood function that photons with indices 1 through $m$ are all from the same, $i$-th intensity state, and $\mathcal{C}$ is a critical value of the test which depends on the number of photons and the confidence interval on which the test is based. Given a minimum signal-to-background ratio of 7 , for instance, change points can be located to within 1-2 photons. In our current analysis, the error rate for misidentifying a true transition is $5 \%$. Whereas the confidence interval for the location of intensity changes is $69 \%$. Intensity values between consecutive change points are extracted using a maximum likelihood estimator, $\hat{I}_{i}=n_{i} / \delta t_{i}$, where $n_{i}$ is number of photons and $\delta t_{i}$ is time interval between change points $(i-1)$ and $i$. The variance associated with this estimate is $\operatorname{var}\left(\hat{I}_{i}\right)=n_{i} /\left(\delta t_{i}\right)^{2}$. This variance will be used later to construct the lifetime-intensity correlation map (cf. section IIIF). In addition to the exact critical values for the test $(\mathcal{C})$ the power of the test, and error rate of this method under different scenarios have been extensively studied using computer simulations [6].

This new method reveals rich dynamics that are otherwise obscured using the popular binning-thresholding method. An example is shown in Fig. 2, where the algorithm identifies a rapid burst at $37.2 \mathrm{~s}$, as well as transitions with subtle changes in intensity (at $\sim 36.5 \mathrm{~s}, \sim 37.5$ $\mathrm{s}$, and $\sim 38.3 \mathrm{~s}$ ), which would have been missed using the traditional visual analysis of binned trajectories.

\section{Maximum likelihood estimation of luminescence decay time}

In addition to emission intensity, each photon contains information pertaining to PL decay rate in its microtime (cf. Fig. ??). To measure excited-sate lifetime with limited number of photons, the maximum likelihood estimator for mono-exponential decays is used [8]. The probability density function for finding a photon at the $j$ th channel given excited-state lifetime $\tau$ in the presence of a constant fraction background has been derived by Köllner and Wolfrum [9]:

$$
p(j)=\frac{b}{k}+(1-b) \frac{\exp (-j r / k)[-1+\exp (r / k)]}{1-\exp (-r)}
$$

where $b$ is fraction of the photon counts that are due to background, $j$ is channel index, $k$ is total number of channels and $r=\tau_{\max } / \tau$, in which $\tau_{\max }$ is the time spanned by $k$ channels. For this experiment, the entire microtime span $\tau_{\max }$ is $342 \mathrm{~ns}$ and the number of channels $k$ included in the analysis is 219 after discarding non-linear regions in 
TCSPC acquisition. The background level for the overall trajectory is estimated from the constant-intensity region before time-zero in an overall luminescence decay curve. For the QD shown in Fig. 2, the background is $\sim 180$ counts per second. Raman scattering only contributes to the bin that corresponds to time-zero and has been subtracted prior to lifetime estimation.

Given a changepoint segment that exhibits an excitedstate lifetime $\tau$ and contains $n$ photons, the distribution of these photons over $k$ microtime channels can be described by the likelihood function, $L(\tau)=\Pi_{i=1}^{n} p(j)$. The maximum likelihood estimator for $\tau$ is the solution to

$$
\frac{\partial}{\partial \tau} \ln L(r)=\frac{\partial r}{\partial \tau}\left[\frac{\partial}{\partial r} \ln L(r)\right]=0 .
$$

Numerical solution to the above equation for QD lifetime was found using the Matlab package. The distribution of a maximum likelihood estimate is asymptotically normal. The variance of the estimate is the inverse of the Fisher information matrix, $J_{F}$,

$$
\operatorname{var}\left(\hat{\tau}_{i}\right)=J_{F}\left(\hat{\tau}_{i}\right)^{-1}=\left\langle\left(\frac{\partial}{\partial \tau} \ln L(\tau)\right)^{2}\right\rangle_{p}^{-1},
$$

where the average $\langle\cdots\rangle_{p}$ is over all microtime channels according to the probability distribution in Eq. (1). This variance will be used later in constructing lifetimeintensity correlation maps (section IIIF).

\section{Autocorrelation analysis of lifetime and intensity fluctuation dynamics}

To assess the amount of dynamic information retained by the changepoint method, normalized autocorrelation functions of intensity or lifetime fluctuation were generated using,

$$
F_{c}(t)=\langle\delta x(t) \delta x(0)\rangle_{T} /\langle x\rangle_{T}^{2}
$$

where $\delta x(t)=x(t)-\langle x\rangle_{T}$, the average $\langle\cdots\rangle_{T}$ is over the entire single QD time trace, and the dynamical variable $x(t)$ could be intensity from the raw photon arrival time data, or intensity from changepoint reconstructed time trajectory, or luminescence lifetime measured from changepoint segments. The statistical errors of the correlation function were calculated following Zwanzig and Ailawadi [10].

* Electronic address: hawyang@berkeley.edu, alivis@ berkeley.edu

[1] S. A. Empedocles and M. G. Bawendi, Science 278, 2114 (1997).

[2] R. G. Neuhauser, K. T. Shimizu, W. K. Woo, S. A. Empedocles, and M. G. Bawendi, Phy. Rev. Lett. 85, 3301 (2000).

[3] X. Chen, N. D. Goorskey, M. Xiao, Z. A. Peng, and X. Peng, Phys. Rev. B 64, 245304 (2001).

[4] W. E. Moerner and D. P. Fromm, Rev. Sci. Inst. 74, 3597 (2003).

[5] H. W. Lilliefors, J. Amer. Stat. Assoc. 64, 387 (1969).

[6] L. P. Watkins and H. Yang, J. Phys. Chem. B 109, 617 (2005).

[7] L. P. Watkins and H. Yang, Biophys. J. 86, 4015 (2004).

[8] P. Hall and B. Selinger, J. Phys. Chem. 85, 2941 (1981).

[9] M. Köllner and J. Wolfrum, Chem. Phys. Lett. 200, 199 (1992).

[10] R. Zwanzig and N. K. Ailawadi, Phys. Rev. 182, 280 (1969). 